\title{
Radioactivity Cross Calibration
}

National Cancer Institute

\section{Source}

National Cancer Institute. Radioactivity Cross Calibration. NCI Thesaurus. Code C94965.

The process of measuring a radioactivity sample in the scanner and a counter. The result is having a consistent measure of radioactivity from these 2 devices when both are used in a protocol. 